\title{
Exponential-Discrete Generalized Exponential Distribution: A New Compound Model
}

\author{
Vahid Nekoukhou \\ Department of Statistics, University of Isfahan, Khansar unit, Isfahan, Iran \\ Corresponding author: v.nekoukhou@gmail.com \\ Hamid Bidram \\ Department of Statistics, University of Isfahan, Isfahan, Iran \\ h.bidram@sci.ui.ac.ir
}

Received 6 May 2015

Accepted 6 April 2016

\begin{abstract}
In this paper, the researchers attempt to introduce a new generalization of the exponential distribution. This new model is obtained by compounding the exponential distribution and discrete generalized exponential distribution of a second type, which is a generalization of the geometric distribution. The new introduced model contains the exponential-geometric distribution as a special case. Some basic distributional properties, moments and order statistics of the new model are discussed. Estimation of the parameters is illustrated, using the maximum likelihood method, and the model with a real data set is also examined.

Keywords: Discrete generalized exponential distribution of a second type; Exponential distribution; Exponential-geometric distribution; Failure rate function; Geometric distribution.
\end{abstract}

2000 Mathematics Subject Classification: 60E05, 62E10

\section{Introduction}

The lifetime of a series (parallel) system with $N$ components is defined by $Y=\min _{1 \leq i \leq N} X_{i}$ $\left(Y=\max _{1 \leq i \leq N} X_{i}\right)$ in which $X_{i}$ denotes the life length of $i$-th $(i=1,2, \ldots, N)$ component. In practice, the number of components itself may be a discrete random variable. In recent years, many researchers have obtained new models to illustrate the characteristics and properties of the lifetime of series and parallel systems. These new models have been appeared in the literature by compounding the known continuous lifetime distributions such as exponential, generalized exponential, gamma and Weibull with classic discrete distributions such as geometric and zero-truncated Poisson, which belong to the family of power series distributions. Indeed, compounding a continuous lifetime distribution with a classic discrete distribution has been widely used by many statisticians to introduce new lifetime models. For example, Adamidis and Loukas (1998) and Kus (2007) introduced the exponential-geometric (EG) and exponential-Poisson distributions, respectively, with 
decreasing failure rates. Tahmasbi and Rezaei (2008) studied the exponential logarithmic distribution. Chahkandi and Ganjali (2009) introduced the family of exponential-power series distributions. As alternative studies in this connection, we can address the Weibull-Poisson distribution proposed by Hemmati et al. (2011) and Lu and Shi (2012), the extended exponential geometric (EEG) distribution of Adamidis et al. (2005) and generalized exponential-power series class of distributions given by Mahmoudi and Jafari (2012). Barreto-Souza et al. (2011) introduced the Weibull-geometric (WG) distribution with decreasing, increasing and upside-down bathtub failure rates. Morais and Barreto-Souza (2011) introduced a compound class of Weibull and power series distributions. The last authors investigated that the failure rate function of the new distributions, similar to WG distribution, is decreasing, increasing and upside-down bathtub.

In this paper, the researchers attempt to introduce another generalization of the exponential distribution. This new model is obtained by compounding the exponential distribution with the discrete generalized exponential (DGE) distribution of a second type introduced by Nekoukhou et al. (2013) with cumulative distribution function (cdf)

$$
F(y ; \gamma, p)=P(Y \leq y)= \begin{cases}0 & y<0 \\ \left(1-p^{[y]+1}\right)^{\gamma} & y \geq 0\end{cases}
$$

A DGE distribution is a generalization of the geometric distribution and depends on two parameters $\gamma>0$ and $0<p<1$. It is interesting to note that a DGE distribution can be viewed as an exponentiated geometric distribution. The probability mass function (pmf) of a DGE distribution, for a non-negative integer $y$, is of the form:

$$
\begin{aligned}
f(y ; \gamma, p)=p_{y}=P(Y=y) & =\left(1-p^{y+1}\right)^{\gamma}-\left(1-p^{y}\right)^{\gamma} \\
& =\sum_{j=1}^{\infty}(-1)^{j+1}\left(\begin{array}{l}
\gamma \\
j
\end{array}\right) p^{j y}\left(1-p^{j}\right),
\end{aligned}
$$

where $\left(\begin{array}{l}\gamma \\ j\end{array}\right)=\frac{\Gamma(\gamma+1)}{\Gamma(\gamma+1-j) j !}$. For an integer value of $\gamma>0$, the sum in Eq. (1.1) stops at $\gamma$. The pmf of the related zero-truncated $D G E(\gamma, p)$ random variable $N$ is also given by

$$
p_{n}=P(N=n)=\frac{\left(1-p^{n+1}\right)^{\gamma}-\left(1-p^{n}\right)^{\gamma}}{1-(1-p)^{\gamma}}, \quad n=1,2,3, \ldots .
$$

If $\gamma=1$, then the DGE distribution and its zero-truncated analog reduce to the known geometric distributions

$$
p_{n}=(1-p) p^{n} \quad n=0,1,2, \ldots
$$

and

$$
p_{n}=(1-p) p^{n-1} \quad n=1,2,3, \ldots
$$

respectively.

In this paper, a new three-parameter lifetime distribution in order to analyze a series system is introduced. Indeed, by considering a series system in which the number of components, $N$, and $X_{i}$ 's follow the DGE and exponential distributions, respectively, we introduce a new generalization of the exponential distribution. This new distribution also contains the EG distribution as a special case. In addition, these new distributions are appropriate models in a supplementary risk problem base 
in presence of latent risks which arise in several areas such as industrial reliability and biomedical studies. Moreover, in the application section of the present paper, we will see that this new generalization of the exponential distribution can give a satisfactory fit in real data analyzing.

The paper is organized as follows: Section 2 introduces a new three-parameter distribution and discusses related sub-models. Section 3 studies some important features and properties of the new model such as the cumulative and failure rate functions, moments, moment generating function and order statistics. In Section 4 the researchers will consider the maximum likelihood estimation of unknown parameters. Section 5 describes the fitting of the proposed model to a well-known real data set. Finally, in Section 6, some concluding remarks are given.

\section{The new distribution}

Since the zero-truncated DGE distribution generalizes the geometric distribution, it is evident to extend the EG distribution by replacing the zero-truncated DGE distribution instead of the geometric distribution in the compounding mechanism.

Suppose that $\left\{X_{i}\right\}_{i=1}^{N}$ are independent and identically distributed (iid) random variables following an exponential distribution with scale parameter $\beta>0$ and probability density function (pdf)

$$
f(x ; \beta)=\beta e^{-\beta x}, \quad x>0,
$$

and $N$, which is independent of $X_{i} s$, is distributed as a zero-truncated $D G E(\gamma, p)$ distribution with $\operatorname{pmf}$ (1.2). The marginal density function of $Y=\min _{1 \leq i \leq N} X_{i}$ is

$$
f(y ; \gamma, p, \beta)=\frac{\beta}{1-(1-p)^{\gamma}} \sum_{n=1}^{\infty} n e^{-n \beta y}\left[\left(1-p^{n+1}\right)^{\gamma}-\left(1-p^{n}\right)^{\gamma}\right],
$$

where $\gamma>0,0<p<1$ and $\beta>0$ are the model parameters. We can find an alternative structure for the pdf of the random variable $Y$, by using Eq. (1.1), as follows:

$$
f(y ; \gamma, p, \beta)=\sum_{j=1}^{\infty} w_{j} f_{E G}\left(y ; p^{j}, \beta\right)
$$

where

$$
w_{j}=\frac{(-1)^{j+1}\left(\begin{array}{l}
\gamma \\
j
\end{array}\right) p^{j}}{1-(1-p)^{\gamma}}, \quad j=1,2, \ldots
$$

and

$$
f_{E G}(y ; p, \beta)=\beta(1-p) e^{-\beta y}\left\{1-p e^{-\beta y}\right\}^{-2}, \quad y>0,
$$

denotes the pdf of the EG distribution of Adamidis and Loukas (1998) with parameters $\beta>0$ and $p<1$. Nekoukhou et al. (2013) investigated that $\sum_{j=1}^{\infty} w_{j}=1$ and if $0<\gamma<1$, then $w_{j}$ 's are positive. Therefore, in this case, the new distribution is an infinite mixture of EG distributions. For integer $\gamma>0$, the sum in Eq. (2.3) stops at $\gamma$.

Some special sub-models of the exponential-discrete generalized exponential (EDGE) distribution, which is defined via Eq. (2.3), are the EG (when $\gamma=1$ ) and the exponential (when $\gamma=1$ and $p \rightarrow 0^{+}$) distributions. When $p \rightarrow 1^{-}$, the EG distribution tends to a distribution degenerate in zero. Hence, an EDGE distribution also contains this special case. 
Remark 2.1 For $|t|<1$ and $k>0$, using the series representation

$$
(1-t)^{-k}=\sum_{i=0}^{\infty} \frac{\Gamma(k+i)}{\Gamma(k) i !} t^{i}
$$

we can expand $\left\{1-p^{j} e^{-\beta y}\right\}^{-2}$ and rewrite the pdf in Eq. (2.3) as

$$
f(y ; \gamma, p, \beta)=\sum_{j=1}^{\infty} \sum_{i=0}^{\infty} w_{j}\left(1-p^{j}\right) p^{i j} f_{E}(y ;(1+i) \beta),
$$

where $f_{E}$ denotes an exponential pdf given by Eq. (2.1).

\section{Basic properties}

\subsection{The distribution and failure rate functions}

The pdf of an EDGE distribution is a linear combination of the EG and exponential distributions via Eq.'s (2.3) and (2.4). Hence, various mathematical properties such as the cdf, moment generating function (mgf) and moments of the EDGE distribution can be obtained from these Eq.'s and the corresponding properties of the EG and exponential distributions.

Let $E D G E(\gamma, p, \beta)$ denote an EDGE distribution with parameters $\gamma, p$ and $\beta$ in the sequel. The cdf of a random variable $Y$ following an $E D G E(\gamma, p, \beta)$ distribution is given by

$$
F(y ; \gamma, p, \beta)=\sum_{j=1}^{\infty} w_{j} F_{E G}\left(y ; p^{j}, \beta\right), \quad y>0
$$

where $F_{E G}$ denotes the cdf of an exponential-geometric distribution. Hence, the cdf of the EDGE distribution is given by

$$
F(y ; \gamma, p, \beta)=\sum_{j=1}^{\infty} w_{j} \frac{1-e^{-\beta y}}{1-p^{j} e^{-\beta y}}, \quad y>0 .
$$

The survival and failure rate functions of $Y$ are given, respectively, by

$$
S(y ; \gamma, p, \beta)=1-\sum_{j=1}^{\infty} w_{j} \frac{1-e^{-\beta y}}{1-p^{j} e^{-\beta y}}, \quad y>0
$$

and

$$
h(y)=\frac{\sum_{j=1}^{\infty} w_{j} f_{E G}\left(y ; p^{j}, \beta\right)}{1-\sum_{j=1}^{\infty} w_{j} \frac{1-e^{-\beta y}}{1-p^{j} e^{-\beta y}}}, \quad y>0 .
$$

Clearly, for integer values of $\gamma>0$, the above infinite sums stop at $\gamma$.

Figure 1 illustrates the shapes of the failure rate function of the EDGE distribution for selected values of its parameters. These plots show that the failure rate function of the EDGE distribution is decreasing. 

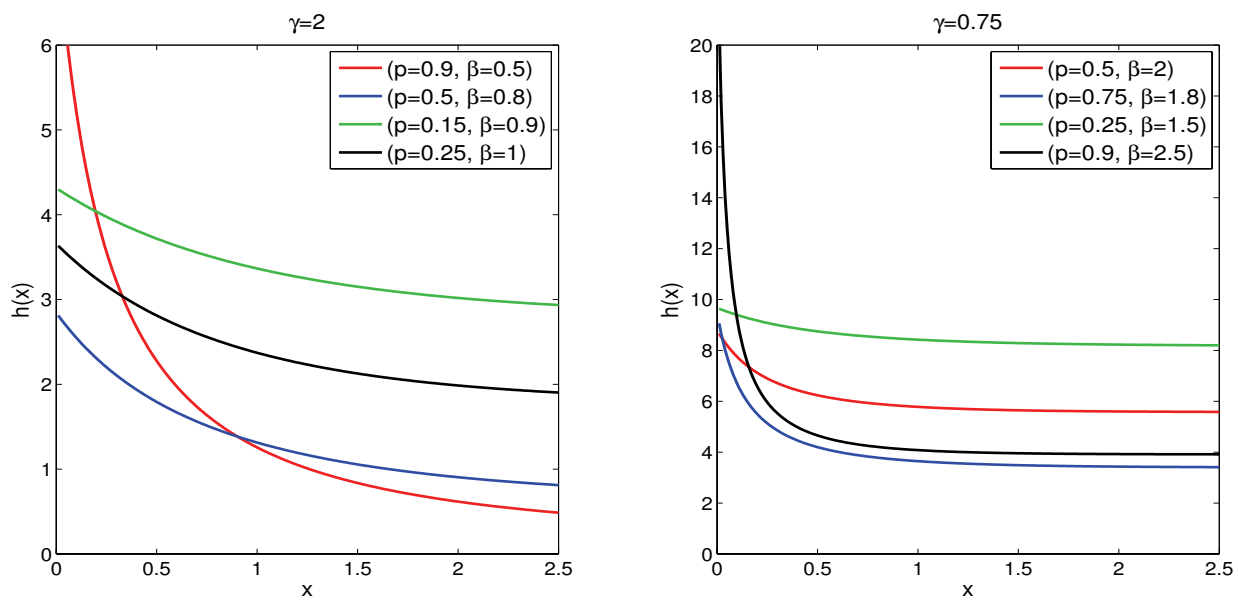

Fig. 1. Failure rate function plots of EDGE distribution for selected parameters values.

\subsection{Moments and moment generating functions}

The $r$-th moment of a random variable $Y \sim E D G E(\gamma, p, \beta)$, by using Eq. (2.3), is given by

$$
E\left(Y^{r}\right)=\sum_{j=1}^{\infty} w_{j} E_{E G}\left(X_{j}^{r}\right), \quad r=1,2, \ldots
$$

where $E_{E G}\left(X_{j}^{r}\right)$ denotes the $r$-th moment of a random variable $X_{j}$ which follows an $E G\left(p^{j}, \beta\right)$ distribution, i.e.,

$$
E_{E G}\left(X_{j}^{r}\right)=\left(1-p^{j}\right) \beta^{-r} \Gamma(r+1) \Phi\left(p^{j}, r, 1\right), \quad j=1,2, \ldots
$$

in which

$$
\Phi(z, s, a)=\{\Gamma(s)\}^{-1} \int_{0}^{\infty} t^{s-1} e^{-a t}\left(1-z e^{-t}\right)^{-1} d t, \quad z<1 ; a, s>0,
$$

is the Lerch's transcendent function (see Erdelyi et al., 1953, pp. 27) and available on MAPLE and MATHEMATICA. One can easily show that $E\left(Y^{r}\right)$ reduces to

$$
E\left(Y^{r}\right)=\frac{\Gamma(r+1)}{\beta^{r}} \sum_{j=1}^{\infty} w_{j} \frac{\left(1-p^{j}\right)}{p^{j}} L\left(p^{j} ; r\right),
$$

where $L(p ; a)=\sum_{t=1}^{\infty} p^{t} t^{-a}$ is Euler's polylogarithm function (see Erdelyi et al., 1953, pp. 31).

Remark 3.1 Bidram et al. (2013) derived an alternative expression for the moments of the $E G(p, \beta)$ distribution as

$$
E_{E G}\left(X^{r}\right)=\frac{(1-p) \Gamma(r+1)}{\beta^{r}} \sum_{i=0}^{\infty} \frac{p^{i}}{(i+1)^{r}}, \quad r=1,2, \ldots
$$

Substituting the above relation into Eq. (3.2) yields another relation for the moments of the EDGE distribution which is given by

$$
E\left(Y^{r}\right)=\frac{\Gamma(r+1)}{\beta^{r}} \sum_{j=1}^{\infty} \sum_{i=0}^{\infty} w_{j} \frac{p^{i j}\left(1-p^{j}\right)}{(i+1)^{r}}, \quad r=1,2, \ldots .
$$


Moreover, the last authors, for the first time, obtained the $\operatorname{mgf}$ of the $E G(p, \beta)$ distribution as

$$
M_{E G}(t)=(1-p) \sum_{i=0}^{\infty} \sum_{k=0}^{\infty} p^{i}\left\{\frac{t}{(i+1) \beta}\right\}^{k} .
$$

Hence, by using Eq.'s (2.3) and (3.4), the mgf of the EDGE distribution is obtained as

$$
M_{Y}(t)=\sum_{j=1}^{\infty} \sum_{i=0}^{\infty} \sum_{k=0}^{\infty} w_{j}\left(1-p^{j}\right) p^{i j}\left\{\frac{t}{(i+1) \beta}\right\}^{k} .
$$

Remark 3.2 Eq. (2.4) is useful for calculating some characteristics of the EDGE distribution from the exponential distribution. For example, the factorial moments generating function of a random variable $Y \sim E D G E(\gamma, p, \beta)$ is given by

$$
\Psi_{Y}(z)=\sum_{j=1}^{\infty} \sum_{t=0}^{\infty} w_{j}\left(1-p^{j}\right) p^{j t} \frac{\Gamma(1+z)}{\beta^{z}(1+t)^{z}} .
$$

Remark 3.3 It is evident that for an integer $\gamma>0, \sum_{j=1}^{\infty}$ should be replaced by $\sum_{j=1}^{\gamma}$ in the above relations.

\subsection{Order statistics}

Order statistics play a key role in both practical and theoretical aspects of Statistics. Specially the importance of the order statistics is shown in non-parametric statistics and statistical inference.

The aim of the present section is to establish the relations regarding the order statistics of the EDGE distribution. More precisely, let $F_{i}(y ; \gamma, p, \beta)$ denote the cdf of the $i$-th order statistic of a random sample $Y_{1}, Y_{2}, \ldots, Y_{n}$ drawn from an $E D G E(\gamma, p, \beta)$ distribution.

Theorem 3.1. The $c d f$ of the $i$-th order statistic of a random sample of size $n$ drawn from an $E D G E(\gamma, p, \beta)$ distribution is given by

$$
F_{i}(y ; \gamma, p, \beta)=\sum_{k=i}^{n} \sum_{j=0}^{n-k} \sum_{m_{1}=1}^{\infty} \ldots \sum_{m_{k+j}=1}^{\infty} \prod_{z=1}^{k+j} \delta_{k, j} F_{E G}\left(y ; p^{m_{z}}, \beta\right)
$$

where

$$
\delta_{k, j}=\frac{\left(\begin{array}{c}
n \\
k
\end{array}\right)\left(\begin{array}{c}
n-k \\
j
\end{array}\right)\left(\begin{array}{c}
\gamma \\
m_{z}
\end{array}\right)(-1)^{\sum_{z=1}^{k+j} m_{z}+k} p^{\sum_{z=1}^{k+j} m_{z}}}{\left[1-(1-p)^{\gamma}\right]^{k+j}},
$$

and the sums in Eq. (3.5) extend over all $(k+j+1)$-tuples $\left(k, m_{1}, m_{2}, \ldots, m_{k+j}\right)$ of non-negative integers.

Proof. Using the binomial expansion for $[1-F(y ; \gamma, p, \beta)]^{n-k}$, one can easily obtain the following representation for the cdf of the $i$-th order statistic,

$$
F_{i}(y ; \gamma, p, \beta)=\sum_{k=i}^{n} \sum_{j=0}^{n-k}\left(\begin{array}{l}
n \\
k
\end{array}\right)\left(\begin{array}{c}
n-k \\
j
\end{array}\right)(-1)^{j}[F(y ; \gamma, p, \beta)]^{k+j} .
$$

Replacing $F(y ; \gamma, p, \beta)$ by Eq. (3.1) yields that

$$
F_{i}(y ; \gamma, p, \beta)=\sum_{k=i}^{n} \sum_{j=0}^{n-k}\left(\begin{array}{c}
n \\
k
\end{array}\right)\left(\begin{array}{c}
n-k \\
j
\end{array}\right)(-1)^{j}\left[\sum_{t=1}^{\infty} w_{t} F_{E G}\left(y ; p^{t}, \beta\right)\right]^{k+j}
$$


In this stage, by using the expansion

$$
\left(\sum_{i=1}^{\infty} a_{i}\right)^{k}=\sum_{m_{1}=1}^{\infty} \sum_{m_{2}=1}^{\infty} \ldots \sum_{m_{k}=1}^{\infty} \prod_{j=1}^{k} a_{m_{j}}, \quad k=1,2, \ldots
$$

Eq. (3.6) can be rewritten as

$$
F_{i}(y ; \gamma, p, \beta)=\sum_{k=i}^{n} \sum_{j=0}^{n-k} \sum_{m_{1}=1}^{\infty} \ldots \sum_{m_{k+j}=1}^{\infty} \prod_{z=1}^{k+j} \delta_{k, j} F_{E G}\left(y ; p^{m_{z}}, \beta\right)
$$

Corollary 3.1. The pdf of the $i$-th order statistic is given by

$$
f_{i}(y ; \gamma, p, \beta)=\sum_{k=i}^{n} \sum_{j=0}^{n-k} \sum_{m_{1}=1}^{\infty} \ldots \sum_{m_{k+j}=1}^{\infty} \prod_{z=1}^{k+j} \delta_{k, j} f_{E G}\left(y ; p^{m_{z}}, \beta\right)
$$

where $f_{E G}$ denotes an EG pdf. Hence, some mathematical properties of the EDGE order statistics can be immediately obtained from Eq. (3.8) and those properties of the EG order statistics.

Remark 3.4 For integer values of $\gamma$, the infinite sums in Eq.'s (3.7) and (3.8) stop at $\gamma$.

Corollary 3.2. The mgf of the $i$-th order statistic of the EDGE distribution, say $M_{i: n}(t)$, can be easily obtained by combining Eq.'s (3.4) and (3.8) as follows:

$$
M_{i: n}(t)=\sum_{k=i}^{n} \sum_{j=0}^{n-k} \sum_{m_{1}=1}^{\infty} \ldots \sum_{m_{k+j}=1}^{\infty} \prod_{z=1}^{k+j} \sum_{l=0}^{\infty} \delta_{k, j}\left(1-p^{m_{z}}\right) p^{l m_{z}} \sum_{r=0}^{\infty}\left\{\frac{t}{(l+1) \beta}\right\}^{r} .
$$

Corollary 3.3. The moments of the i-th order statistic are derived by combining Eq.'s (3.3) and (3.8) which are given by

$$
E\left(Y_{i: n}^{r}\right)=\sum_{k=i}^{n} \sum_{j=0}^{n-k} \sum_{m_{1}=1}^{\infty} \ldots \sum_{m_{k+j}=1}^{\infty} \prod_{z=1}^{k+j} \sum_{i=0}^{\infty} \delta_{k, j} \frac{\left(1-p^{m_{z}}\right) \Gamma(r+1)}{\beta^{r}} \frac{p^{i m_{z}}}{(i+1)^{r}}, \quad r=1,2, \ldots
$$

Remark 3.5 We can show that when $\gamma=1$, Eq. (3.9) reduces to

$$
E\left(Y_{i: n}^{r}\right)=\frac{\Gamma(r+1)(1-p)}{B(i, n-i+1) \beta^{r}} \sum_{k=0}^{n-i} \sum_{h=0}^{\infty} \sum_{j=0}^{k+i-1} \frac{\left(\begin{array}{c}
n-i \\
k
\end{array}\right)\left(\begin{array}{c}
k+i+h \\
h
\end{array}\right)\left(\begin{array}{c}
k+i-1 \\
j
\end{array}\right)(-1)^{k+j} p^{h}}{(1+h+j)^{r+1}},
$$

which is the $r$-th moment of the $i$-th order statistic of an $E G(p, \beta)$ distribution derived by Bidram et al. (2013).

\subsection{Rényi entropy}

The entropy of a random variable $Y$ is a measure of uncertainty variation. The Rényi entropy is important in Ecology, Statistics as indices of diversity, Reliability and also important in quantum 
information, which it can be used as a measure of entanglement. Rényi entropy is defined by

$$
I_{R}(\rho)=\frac{1}{1-\rho} \log \left\{\int_{\mathscr{R}} f^{\rho}(y) d y\right\}
$$

where $\rho>0$ and $\rho \neq 1$; see Rényi (1961). For the EDGE pdf given by (2.3), we see that

$$
\int_{0}^{\infty} f^{\rho}(y ; \gamma, p, \beta) d y=\int_{0}^{\infty}\left[\sum_{j=1}^{\infty} w_{j} f_{E G}\left(y ; p^{j}, \beta\right)\right]^{\rho} d y .
$$

Using the series representation (3.7), it is easy to show that

$$
I_{R}(\rho)=\frac{1}{1-\rho} \log \frac{1}{\left[1-(1-p)^{\gamma}\right] \rho} \sum_{m_{1}=1}^{\infty} \sum_{m_{2}=1}^{\infty} \ldots \sum_{m_{\rho}=1}^{\infty} \prod_{j=1}^{\rho}(-1)^{m_{j}+1}\left(\begin{array}{c}
\gamma \\
m_{j}
\end{array}\right) p_{j}^{m} .
$$

As mentioned before, the above infinite sums stop at $\gamma$ for integer values of $\gamma$.

\section{Estimation}

To apply the method of maximum likelihood for estimating the unknown parameters vector $\theta=$ $(\gamma, p, \beta)^{T}$ of an EDGE distribution, assume that $\mathbf{y}=\left(y_{1}, y_{2}, \ldots, y_{m}\right)^{T}$ is a random sample of size $m$ from the distribution. The log-likelihood function, using Eq. (2.2), becomes

$$
\ell=m \log \beta-m \log \left[1-(1-p)^{\gamma}\right]+\sum_{i=1}^{m} \log \left(\sum_{n=1}^{\infty} n e^{-n \beta y_{i}}\left[\left(1-p^{n+1}\right)^{\gamma}-\left(1-p^{n}\right)^{\gamma}\right]\right) .
$$

Hence, the likelihood equations are

$$
\begin{aligned}
\frac{\partial \ell}{\partial \gamma} & =\sum_{i=1}^{m} \frac{\sum_{n=1}^{\infty} n e^{-n \beta y_{i}}\left[\left(1-p^{n+1}\right)^{\gamma} \log \left(1-p^{n+1}\right)-\left(1-p^{n}\right)^{\gamma} \log \left(1-p^{n}\right)\right]}{\sum_{n=1}^{\infty} n e^{-n \beta y_{i}}\left[\left(1-p^{n+1}\right)^{\gamma}-\left(1-p^{n}\right)^{\gamma}\right]} \\
& +\frac{m(1-p)^{\gamma} \log (1-p)}{1-(1-p)^{\gamma}} \\
\frac{\partial \ell}{\partial p} & =\sum_{i=1}^{m} \frac{\sum_{n=1}^{\infty} n e^{-n \beta y_{i}}\left[n \gamma p^{n-1}\left(1-p^{n}\right)^{\gamma-1}-(n+1) \gamma p^{n}\left(1-p^{n+1}\right)^{\gamma-1}\right]}{\sum_{n=1}^{\infty} n e^{-n \beta y_{i}}\left[\left(1-p^{n+1}\right)^{\gamma}-\left(1-p^{n}\right)^{\gamma}\right]} \\
& -m \frac{\gamma(1-p)^{\gamma-1}}{1-(1-p)^{\gamma}}
\end{aligned}
$$

and

$$
\frac{\partial \ell}{\partial \beta}=\sum_{i=1}^{m} \frac{\sum_{n=1}^{\infty}-n^{2} e^{-n \beta y_{i}} \beta y_{i}\left[\left(1-p^{n+1}\right)^{\gamma}-\left(1-p^{n}\right)^{\gamma}\right]}{\beta \sum_{n=1}^{\infty} n e^{-n \beta y_{i}}\left[\left(1-p^{n+1}\right)^{\gamma}-\left(1-p^{n}\right)^{\gamma}\right]}
$$

The maximum likelihood estimate (MLE) of $\theta$, say $\hat{\theta}$, can be obtained by solving the nonlinear equation $(\partial \ell / \partial \gamma, \partial \ell / \partial p, \partial \ell / \partial \beta)^{T}=0$ using a numerical method such as the Newton-Raphson algorithm. Under the regular conditions, stated in Cox and Hinkley (1974), that are fulfilled for the parameter $\theta$ in the interior of the parameter space but not on the boundary, the MLE vector $\hat{\theta}$ is consistent and the asymptotic distribution of $I_{\mathbf{y}}^{\frac{1}{2}}(\theta)(\hat{\theta}-\theta)$ is a multivariate normal with the (vector) mean zero and the identity covariance matrix in which $I_{\mathbf{y}}(\theta)$ is the information matrix. 
The observed information matrix, which is used for interval estimation and hypotheses testing on the model parameters, is given by

$$
I_{\mathbf{y}}(\hat{\theta})=\left[\begin{array}{ccc}
-\frac{\partial^{2} \ell}{\partial \gamma^{2}} & -\frac{\partial^{2} \ell}{\partial \gamma \partial p} & -\frac{\partial^{2} \ell}{\partial \gamma \partial \beta} \\
-\frac{\partial^{2} \ell}{\partial p \partial \gamma} & -\frac{\partial^{2} \ell}{\partial p^{2}} & -\frac{\partial^{2} \ell}{\partial p \partial \beta} \\
-\frac{\partial^{2} \ell}{\partial \beta \partial \gamma} & -\frac{\partial^{2} \ell}{\partial \beta \partial p} & -\frac{\partial^{2} \ell}{\partial \beta^{2}}
\end{array}\right]_{\left.\right|_{\theta=\hat{\theta}}} .
$$

One can use the normal distribution of $\hat{\theta}$ to construct approximate confidence regions for some parameters and for the failure and survival functions. Indeed, an asymptotic $100(1-\xi)$ confidence interval for each parameter $\theta_{i}$, is given by

$$
\left(\hat{\theta}_{i}-z_{\xi / 2} \sqrt{\hat{J}_{i i}}, \hat{\theta}_{i}+z_{\xi / 2} \sqrt{\hat{J}_{i i}}\right), \quad i=1,2,3,
$$

where $\hat{J}_{i i}$ denotes the $(i, i)$ diagonal element of $I_{\mathbf{y}}^{-1}(\hat{\theta})$ and $z_{\xi / 2}$ is the $(1-\xi / 2)$-th quantile of the standard normal distribution.

\section{Application}

In order to illustrate the capacity of EDGE distributions in data modeling, the Boing data which have been used widely in the literature are considered. This data set consists of the number of successive failures of the air conditioning system of each number of a fleet of 13 Boeing 720 jet airplanes. The pooled data with 213 observations, first analyzed by Proschan (1963) and discussed further by Adamidis and Loukas (1998) and also Bidram et al. (2013).

In order to identify the shape of the hazard rate function of these data, we considered a graphical method based on the Total Time on Test (TTT) plot. As we know, the empirical TTT plot is given by

$$
G(r / n)=\left(\sum_{i=1}^{r} Y_{i: n}+(n-r) Y_{r: n}\right) / \sum_{i=1}^{n} Y_{i: n}, \quad r=1,2, \ldots, n,
$$

where $Y_{i: n}$ denotes the $i$-th order statistic of the sample. If the empirical TTT transform is convex, concave, convex then concave and concave then convex, the shape of the corresponding hazard rate function is, respectively, decreasing, increasing, bathtub-shaped and upside-down bathtub; see, e.g., Aarset (1987). Figure 2 shows the TTT plot of this data set. As we see from the figure, the hazard rate of the data set is decreasing.

Here, the three-parameter exponentiated Weibull (EW) distribution of Mudholkar and Srivastava (1993) and Weibull-geometric distribution of Barreto-Souza et al. (2011), whose hazard rate functions can be decreasing, are compared with the EDGE distribution. Also, the EG distribution as a sub-model of the EDGE distribution is fitted. Table 1 indicates the related fitting computations which consists of the MLEs, Akaike information criterions (AICs) and values of KolmogorovSmirnov (K-S) test statistics with the corresponding p-values.

Table 1. MLEs, AICs and K-S test statistics (p-values) for the data set.

\begin{tabular}{lccc}
\hline Model & MLEs of parameters & AIC & K-S (p-value $)$ \\
\hline EDGE & $(\hat{\gamma}, \hat{\beta}, \hat{p})=(11.0921,0.0023,0.5606)$ & 2304.1 & $0.0361(0.9116)$ \\
WG & $(\hat{\alpha}, \hat{\beta}, \hat{p})=(1.2246,0.0048,0.7841)$ & 2354.3 & $0.0395(0.8812)$ \\
EW & $(\hat{\gamma}, \hat{\beta}, \hat{\alpha})=(2.5923,0.0344,0.5778)$ & 2355.2 & $0.0379(0.9084)$ \\
EG & $(\hat{\beta}, \hat{p})=(0.0081,0.4276)$ & 2355.9 & $0.0508(0.6228)$ \\
\hline
\end{tabular}




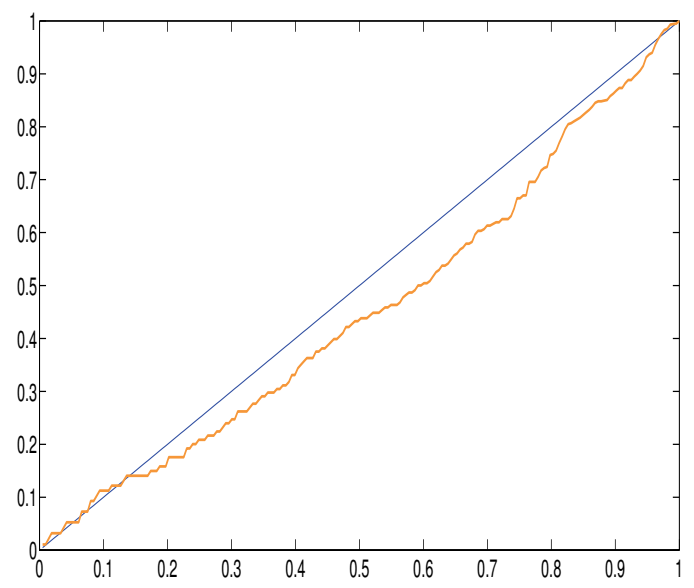

Fig. 2. Empirical TTT plot of Boing data.

According to the AIC values of fitted models, given in Table 1, we find that the EDGE distribution gives a better fit than other rival models. In addition, the p-values of the K-S test statistics confirm this claim. According to the results given in Table 1 and Figures 3 and 4, it seems that the EDGE distribution provides a satisfactory fit to this real data set.

One can construct approximate confidence intervals for the parameters of the EDGE model. Indeed, such confidence intervals are attained by means of the asymptotic covariance matrix of the MLEs of EDGE parameters when the Newton-Raphson procedure converges in, e.g., MATLAB software. For instance, $95 \%$ asymptotic confidence intervals for the parameters are calculated as $\gamma \in(11.0921 \mp 0.0368), \beta \in(0.0023 \mp 0.0005)$ and $p \in(0.5606 \mp 0.1012)$.

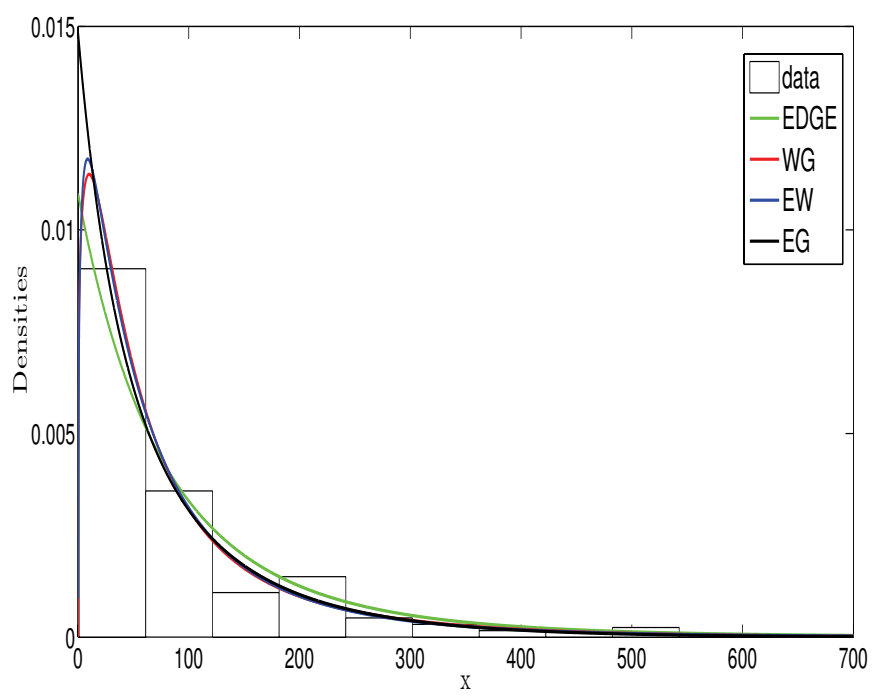

Fig. 3. Densities plot of the fitted models 

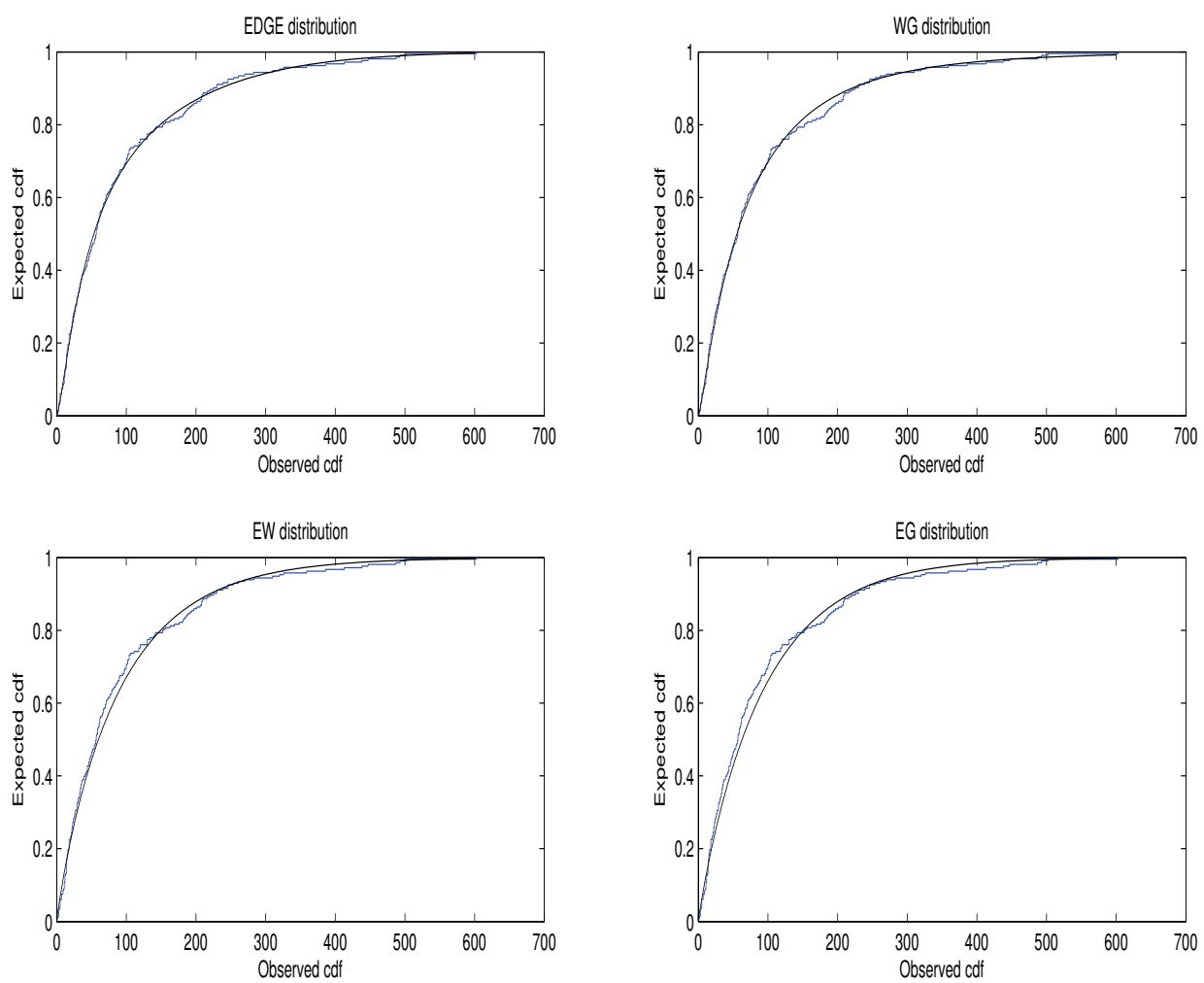

Fig. 4. Empirical cdf plots of the fitted models

\section{Concluding remarks}

In this paper, a new three-parameter generalization of the exponential and exponential-geometric distributions is proposed, so-called exponential-discrete generalized exponential (EDGE) distribution. The failure rate function of the new model is decreasing. Some important probabilistic properties and the problem of estimation of its parameters are studied. Additionally, EDGE distributions can provide a satisfactory parametric fit to some real data sets.

\section{Acknowledgments}

The authors sincerely thank the anonymous referees and the Associate Editor for their valuable considerations.

\section{References}

[1] M.V. Aarset, How to identify a bathtub hazard rate, IEEE Trans. Rel. 36 (1987) 106-108.

[2] K. Adamidis, T. Dimitrakopoulou and S. Loukas, On an extension of the exponential-geometric distribution, Stat. Prob. Lett. 73 (2005) 259-269.

[3] K. Adamidis and S. Loukas, A lifetime distribution with decreasing failure rate, Stat. Prob. Lett. 39 (1998) 35-42.

[4] W. Barreto-Souza, A.L. de Morais and G.M. Cordeiro, The Weibull-geometric distribution, J. Stat. Comput. Simul. 81 (2011) 645-657.

[5] H. Bidram, J. Behboodian and M. Towhidi, The beta Weibull-geometric distribution, J. Stat. Comput. Simul. 83(1) (2013) 52-67. 
[6] M. Chahkandi and M. Ganjali, On some lifetime distributions with decreasing failure rate, Comput. Stat. Data Anal. 53 (2009) 4433-4440.

[7] D.R. Cox and D.V. Hinkley, Theoretical Statistics, (Chapman and Hall, London, 1974).

[8] A. Erdelyi, W. Magnus, F. Oberhettinger and F.G. Tricomi, Higher Transcendental Functions, (McGraw-Hill, New York, 1953).

[9] F. Hemmati, E., Khorram and S. Rezakhah, A new three-parameter ageing distribution, J. Stat. Plann. Inf. 141 (2012) 2266-2275.

[10] C. Kus, A new lifetime distribution, Comput. Stat. Data Anal. 51 (2007) 4497-4509.

[11] W. Lu and D. and Shi, A new compounding life distribution: the Weibull-Poisson distribution, J. Appl. Stat. 39(1) (2012) 21-38.

[12] E. Mahmoudi and A.A. Jafari, Generalized exponential-power series distributions, Comput. Stat. Data Anal. 56 (2012) 4047-4066.

[13] A.L. Morais and W. Barreto-Souza, A compound class of Weibull and power series distributions, Comput. Stat. Data Anal. 55 (2011) 1410-1425.

[14] G.S. Mudholkar and D.K. Srivastava, Exponentiated Weibull family for analyzing bathtub failure-real data, IEEE Trans. Reliab. 42 (1993) 299-302.

[15] V. Nekoukhou, M.H. Alamatsaz and H. Bidram, Discrete generalized exponential distribution of a second type, Statistics. 47(4) (2013) 876-887.

[16] F. Proschan, Theoretical explanation of observed decreasing failure rate, Technometrics. 5 (1963) 375383.

[17] A. Rényi, On measures of entropy and information, In: Proceedings of the fourth Berkeley sympsium on mathematical statistics and probability, Berkeley: University of California Press. 1 (1961) 547-561.

[18] R. Tahmasbi and S. Rezaei, A two-parameter lifetime distribution with decreasing failure rate, Comput. Stat. Data Anal. 52 (2008) 3889-3901. 